\title{
FREE RIGHT TYPE A SEMIGROUPS
}

\section{by JOHN FOUNTAIN}

(Received 10 October, 1989)

Introduction. The relation $\mathscr{L}^{*}$ is defined on a semigroup $S$ by the rule that $a \mathscr{L}^{*} b$ if and only if the elements $a, b$ of $S$ are related by Green's relation $\mathscr{L}$ in some oversemigroup of $S$. A semigroup $S$ is an $E$-semigroup if its set $E(S)$ of idempotents is a subsemilattice of $S$. A right adequate semigroup is an $E$-semigroup in which every $\mathscr{L}^{*}$-class contains an idempotent. It is easy to see that, in fact, each $\mathscr{L}^{*}$-class of a right adequate semigroup contains a unique idempotent [8]. We denote the idempotent in the $\mathscr{L}^{*}$-class of $a$ by $a^{*}$. Then we may regard a right adequate semigroup as an algebra with a binary operation of multiplication and a unary operation *. We will refer to such algebras as *-semigroups. In [10], it is observed that viewed in this way the class of right adequate semigroups is a quasi-variety.

In this paper, which is the promised sequel to [10], we are concerned with right type A semigroups. These are semigroups which are right adequate and in which $e a=a(e a)^{*}$ for each $a \in S$ and $e \in E(S)$; they form a sub-quasi-variety of the quasi-variety of all right adequate semigroups. Thus, from general results in universal algebra, we know that free right type A semigroups exist. It is the purpose of this paper to give an explicit description of these free objects and to discuss some of their properties.

Our approach is to make use of the construction of free right h-adequate semigroups in [10], a right adequate semigroup being right $h$-adequate when the mapping $\alpha_{a}: E(S)^{1} \rightarrow E(S)^{1}$ defined by $x \alpha_{a}=(x a)^{*}$ is a homomorphism for each element $a$ of $S$. By $a^{*}$-congruence on a right adequate semigroup $S$, we mean a congruence on $S$ regarded as a *-semigroup, that is, a semigroup congruence $\rho$ on $S$ which also satisfies $a \rho b$ implies $a^{*} \rho b^{*}$. A *-congruence $\rho$ on a right adequate semigroup $S$ is called a right type $A$ congruence if $S / \rho$ is a right type A semigroup, where the semigroup $S / \rho$ is made into a ${ }^{*}$-semigroup by defining $(a \rho)^{*}$ to be $a^{*} \rho$. On any right adequate semigroup, there is a minimum right type $A$ congruence and if $\gamma$ is this congruence on $P_{X}$, the free right h-adequate semigroup on $X$, then $P_{X} / \gamma$ is the free right type A semigroup on $X$. For any non-empty set $X$, we construct a semigroup $A_{X}$ isomorphic to $P_{X} / \gamma$ which is analogous to Scheiblich's construction [20] of the free inverse semigroup on $X$.

This construction allows us to obtain several results which are analogues of theorems on inverse semigroups. For example, the fact that free inverse semigroups are $E$-unitary gives rise to one proof that every inverse semigroup has an $E$-unitary cover [18, Theorem VIII.1.10]. The corresponding result for right type A semigroups is that every right type A semigroup has a proper cover [8]. On a right type A semigroup, the minimum left cancellative congruence is denoted by $\sigma$ and the semigroup is proper if $\sigma \cap \mathscr{L}^{*}=\imath$. It is easily seen that $A_{X}$ is proper and we give a new proof of the covering result modelled on that in the inverse case.

After recalling the basic properties of right type A semigroups in Section 1, we devote Section 2 to the construction outlined above. In Section 3, we show that $A_{X}$ enjoys properties similar to those of $P_{X}$. Among other things we have that Green's relations on $A_{X}$ are trivial, the word problem for $A_{X}$ is solvable and $A_{X}$ is residually finite.

We examine sets of free generators in right type $A$ semigroups in Section 4 . The

Glasgow Math. J. 33 (1991) 135-148. 
results are similar to those of Section 4 in [10] and both sets of results are inspired by those of Reilly [19] in the inverse case.

In the final section we obtain a simple description of the free objects in the quasi-variety of adequate semigroups with central idempotents. This parallels the description of free Clifford semigroups in Chapter VIII of [18].

1. Preliminaries. We begin by giving some elementary facts about right adequate and right type A semigroups. Alternative characterisations of the relation $\mathscr{L}^{*}$ are given by the following lemma from [14] and [17].

Lemma 1.1. Let $S$ be a semigroup and let $a, b$ be elements of $S$. Then the following conditions are equivalent:

(1) $a \mathscr{L}^{*} b$,

(2) for all $x, y \in S^{1}, a x=a y$ if and only if $b x=b y$,

(3) there is an $S^{1}$-isomorphism $\phi: a S^{1} \rightarrow b S^{1}$ with $a \phi=b$.

As an easy consequence we have the following corollary.

Corollary 1.2. If $e$ is an idempotent of a semigroup $S$ then the following are equivalent for an element a of $S$ :

(1) $e \mathscr{L}^{*} a$,

(2) $a e=a$ and, for all $x, y$ in $S^{1}, a x=$ ay implies $e x=e y$.

From the definition and Lemma 1.1, it follows that $\mathscr{L}^{*}$ is a right congruence and that $\mathscr{L} \subseteq \mathscr{L}^{*}$. It is well known and easy to see that, for regular elements $a, b$ of $S$, we have $a \mathscr{L}^{*} b$ if and only if $a \mathscr{L} b$. In particular, if $S$ is a regular semigroup then $\mathscr{L}=\mathscr{L}^{*}$.

We record next some elementary properties of right adequate semigroups which we use repeatedly.

Proposition 1.3 [9, Proposition 1.6]. Let $S$ be a right adequate semigroup and $a, b$ elements of $S$. Then

(1) $a \mathscr{L}^{*} b$ if and only if $a^{*}=b^{*}$,

(2) $(a b)^{*}=\left(a^{*} b\right)^{*}$,

(3) $(a b)^{*} \leqslant b^{*}$, where $\leqslant$ is the natural ordering on the semilattice $E(S)$.

As noted in [9, Lemma 2.1], if $S$ is a right type A semigroup then $(e f a)^{*}=(e a)^{*}(f a)^{*}$ for all $e, f \in E(S), a \in S$. Thus, in our present terminology, a right type A semigroup is right h-adequate.

From universal algebra, we have the notions of ${ }^{*}$-subsemigroup, ${ }^{*}$-homomorphism and ${ }^{*}$-congruence. It is clear that if $S$ is right adequate, right $\mathrm{h}$-adequate or right type $\mathrm{A}$ then so is any ${ }^{*}$-subsemigroup of $S$. A left ${ }^{*}$-ideal of $S$ is a left ideal of $S$ which is also a *-subsemigroup of $S$. By a *-ideal of $S$, we mean an ideal of $S$ which is also a left *-ideal. In the case of a right adequate semigroup $S$, a left ideal $I$ is a left ${ }^{*}$-ideal if and only if it is a union of $\mathscr{L}^{*}$-classes.

These ideas are connected by the following result from [10].

Proposition 1.4. Let $S$ be a right adequate semigroup and let I be ${ }^{*}{ }^{*}$-ideal of $S$. Then the Rees quotient semigroup $S / I$ is right adequate and the natural map $v: S \rightarrow S / I$ is a *-homomorphism. Furthermore, if $S$ is right h-adequate or right type $A$ then so is $S / I$. 
Let $\rho$ be a *-congruence on a right type A semigroup $S$. We conclude this section by considering the smallest *-congruence on $S$ which induces the same partition of $E(S)$ as $\rho$. It is a relatively straightforward adaptation of the corresponding result for inverse semigroups [18]. We define the relation $\rho_{\min }$ on $S$ by

$$
a \rho_{\min } b \text { if and only if } a e=b e \text { for some } e \in E(S) \text { with } e \rho a^{*} \rho b^{*} \text {. }
$$

Proposition 1.5. Let $\rho$ be a ${ }^{*}$-congruence on a right type A semigroup $S$. Then $\rho_{\min }$ is $a^{*}$-congruence on $S, \rho_{\min }|E(S)=\rho| E(S)$ and $\rho_{\min } \subseteq \tau$ for any ${ }^{*}$-congruence $\tau$ on $S$ with $\tau|E(S)=\rho| E(S)$. Furthermore, $S / \rho_{\min }$ is right type $A$ and

$$
E\left(S / \rho_{\min }\right)=\left\{e \rho_{\min }: e \in E(S)\right\} .
$$

Proof. Clearly $\rho_{\min }$ is reflexive and symmetric. If $a \rho_{\min } b, b \rho_{\min } c$ then there are idempotents $e, f$ with $e \rho a^{*} \rho b^{*} \rho c^{*} \rho f, a e=b e$ and $b f=c f$. Hence $e^{2} \rho e f$; so that $e f \rho a^{*} \rho c^{*}$ and $a e f=b e f=c e f$, whence $a \rho_{\min } c$.

Now let $a \rho_{\min } b$ and $c \in S$. Then $a e=b e$ for some idempotent $e$ with $e \rho a^{*} \rho b^{*}$. Certainly $(c a) e(c a)^{*}=(c b) e(c a)^{*}$ and, from Proposition 1.3,

$$
\begin{aligned}
& (c a)^{*}=a^{*}(c a)^{*} \rho e(c a)^{*}, \\
& (c b)^{*}=b^{*}(c b)^{*}=b^{*}(c b)^{*} b^{*} \rho e(c b)^{*} e .
\end{aligned}
$$

Now $c a e=c b e$ so that $(c a)^{*} e=(c a e)^{*}=(c b e)^{*}=(c b)^{*} e$ and hence

Thus $c a \rho_{\min } c b$.

$$
(c b)^{*} \rho e(c a)^{*} e=e(c a)^{*} \text {. }
$$

Since $S$ is right type A, we have $e c=c(e c)^{*}$; so that

$$
(a c)(e c)^{*}=a e c=b e c=(c)(e c)^{*} \text {. }
$$

As $a^{*} \rho e$, we have $a^{*} c \rho e c$ and so $\left(a^{*} c\right)^{*} \rho(e c)^{*}$ since $\rho$ is a ${ }^{*}$-congruence. But $\left(a^{*} c\right)^{*}=(a c)^{*}$; so that $(a c)^{*} \rho(e c)^{*}$. Similarly $(b c)^{*} \rho(e c)^{*}$, and hence $a c \rho_{\min } b c$.

Also, from $a e=b e$, we obtain

$$
a^{*} e=(a e)^{*}=(b e)^{*}=b^{*} e
$$

and, as $a^{*} \rho b^{*} \rho e$, we conclude that $a^{*} \rho_{\min } b^{*}$.

Thus $\rho_{\min }$ is a ${ }^{*}$-congruence on $S$.

If $e, f \in E(S)$ and $e \rho f$ then $e \rho e f$ and $e(e f)=f(e f)$; so that $e \rho_{\min } f$. Conversely, if $e \rho_{\min } f$ then it is immediate that $e \rho f$ and thus $\rho\left|E(S)=\rho_{\min }\right| E(S)$.

Now let $\tau$ be a ${ }^{*}$-congruence on $S$ with $\rho|E(S)=\tau| E(S)$. If $a \rho_{\min } b$ then $a e=b e$ for some idempotent $e$ with $e \rho a^{*} \rho b^{*}$. Thus $e \tau a^{*} \tau b^{*}$ and so $a a^{*} \tau a e, b b^{*} \tau b e$, whence $a a^{*} \tau b b^{*}$, that is, $a \tau b$. Thus $\rho_{\min } \subseteq \tau$.

To show that the ${ }^{*}$-semigroup $S / \rho_{\min }$ is right type $\mathrm{A}$, it is sufficient to show that it is right adequate. To see this, let $a \in S, x, y \in S^{1}$ and suppose that $a x \rho_{\min } a y$. Then axe =aye for some $e \in E(S)$ with $(a x)^{*} \rho(a y)^{*} \rho e$. Since $a^{*} \mathscr{L}^{*} a$, we thus have $a^{*} x e=a^{*} y e$ and, by Proposition 1.3, $\left(a^{*} x\right)^{*} \rho\left(a^{*} y\right)^{*} \rho e$; so that $a^{*} x \rho_{\min } a^{*} y$ as required and $S / \rho_{\min }$ is right type A.

If $a^{2} \rho_{\min } a$ then, from above, $a^{*} a \rho_{\min } a^{*}$ and hence $\left(a^{*} a\right)^{*} \rho_{\min } a^{*}$. Thus $e \rho_{\min } a^{*}$, where $e=a^{*}\left(a^{*} a\right)^{*}$. Now $e=\left(a\left(a^{*} a\right)^{*}\right)^{*}$ and $\rho_{\min } \subseteq \rho$; so that, from $a e=a\left(a^{*} a\right)^{*} e$, we conclude $a \rho_{\min } a\left(a^{*} a\right)^{*}$. But $a^{*} a=a\left(a^{*} a\right)^{*}$ since $S$ is right type $\mathrm{A}$ and so $a \rho_{\min } a^{*}$. It follows that the set of idempotents of $S / \rho_{\min }$ is $\left\{e \rho_{\min }: e \in E(S)\right\}$. 
By considering the case when $\rho=\omega$ is the universal congruence on $S$, it is straightforward to obtain the following corollary which is Lemma 1.3 of [8] (see also [12, Proposition 1.7]). We denote $\omega_{\min }$ by $\sigma$ and note that $a \sigma b$ if and only if $a e=b e$ for some idempotent $e$ of $S$.

Corollary 1.6. Let $S$ be a right type A semigroup. Then $\sigma$ is a ${ }^{*}$-congruence on $S$, $S / \sigma$ is a left cancellative monoid and $\sigma$ is the minimum left cancellative congruence on $S$.

Following [8], we define a right type A semigroup to be proper if $\sigma \cap \mathscr{L}^{*}=\iota$. Every proper right type A semigroup $S$ is $E$-unitary (i.e. $a \in S$, ae, $e \in E(S)$ implies $a \in E(S)$ ) but the converse is not true as shown by Example 3 of [8].

Proposition 1.7. If $\rho$ is $a^{*}$-congruence on a proper right type $A$ semigroup $S$ then $S / \rho_{\min }$ is proper.

Proof. Write $\tau$ for $\rho_{\min }$ and suppose that $a \tau, b \tau$ are $\left(\sigma \cap \mathscr{L}^{*}\right)$-related in $S / \tau$. Then $(\mathrm{a} \tau)^{*}=(b \tau)^{*}$ so that $a^{*} \tau b^{*}$ and hence $a^{*} f=b^{*} f$ for some $f \in E(S)$ with $f \rho a^{*} \rho b^{*}$.

Now $(a \tau)(e \tau)=(b \tau)(e \tau)$ for some $e \in E(S)$ since $a \tau, b \tau$ are $\sigma$-related in $S / \tau$. Thus $a e \tau b e$ and so $a e h=b e h$ for some idempotent $h$. This gives $a \sigma b$ and so afobf. But $(a f)^{*}=a^{*} f=b^{*} f=(b f)^{*}$ so that $a f \mathscr{L}^{*} b f$ and, as $S$ is proper, we get $a f=b f$. But $f \rho a^{*} \rho b^{*}$ and so $a \tau b$ as required and $S / \tau$ is proper.

2. The semigroup $A_{X}$. In this section, we construct the free right type A semigroup $A_{X}$ on a set $X$. We show that $A_{X}$ is free by showing that it is isomorphic to $P_{X} / \gamma$, where $P_{X}$ is the free right h-adequate semigroup on $X$ and $\gamma$ is the minimum right type A congruence on $P_{X}$. We begin by recalling the construction of $P_{X}$ in [10].

Let $F_{X}$ be the free semigroup on $X$ and partially order $F_{X}$ by putting $u \leqslant v$ if and only if $u$ is a final segment of $v$. For any subset $A$ of $F_{X}$, we write

$$
\max A=\{a \in A: a \text { is maximal in } A \text { under } \leqslant\} .
$$

Now let

$$
E_{X}=\left\{A: A \subseteq F_{X}, A \text { is finite and non-empty, } A=\max A\right\} .
$$

Thus $E_{X}$ is the set of all finite suffix codes over $X$. For $A, B \in E_{X}$, let $A B=\max (A \cup B)$. Then $E_{X}$ is a semilattice; in fact, if we consider $F_{X}$ as partially ordered by the dual of the above ordering then $E_{X}$ is the free semilattice on this partially ordered set [11]. We note that the following statements are equivalent for members $A, B$ of $E_{X}$ where we use $\leqslant$ for the order relation in $E_{X}$ as well as that in $F_{X}$ :

$A \leqslant B ; A B=A ; \max (A \cup B)=A$; for each $b$ in $B$, there is an $a$ in $A$

such that $b \leqslant a$; each element in $B$ is a final segment of some

element in $A$.

For $w \in F_{X}, A \in E_{X}$, we put $A . w=\{a w: a \in A\}$. Clearly $A, w \in E_{X}$ and we have an action of $F_{X}$ on $E_{X}$. Furthermore, if $w \in F_{X}, A, B \in E_{X}$ then it is routine to verify that

$$
(A B) \cdot w=(A \cdot w)(B \cdot w)
$$

and consequently the action is order-preserving.

For each element $w$ of $F_{X}$, we define $w^{*}$ to be the singleton $\{w\} \in E_{X}$. We note that if $A=\left\{w_{1}, \ldots, w_{k}\right\} \in E_{X}$ then $A=w_{1}^{*} \ldots w_{k}^{*}$; so that $E_{X}$ is generated by the set $\left\{w^{*}: w \in F_{X}\right\}$. We also observe that, for any $A \in E_{X}, w \in F_{X}$, we have $A . w \leqslant\{w\}=w^{*}$. 
Consider the free product $F_{X} * E_{X}$ in the category of semigroups. Its elements can be written uniquely as words $a=s_{1} \ldots s_{n}$, where $s_{i} \in F_{X} \cup E_{X}$ and, for $i=1, \ldots, n-1$, the elements $s_{i}, s_{i+1}$ are not both in the same factor $F_{X}$ or $E_{X}$.

We extend ${ }^{*}$ from $F_{X}$ to $F_{X} * E_{X}$ as follows: for $A \in E_{X}$, we put $A^{*}=A$ and if $a=s_{1} \ldots s_{n}$ (as above), $b=s_{1} \ldots s_{n-1}$ and $b^{*} \in E_{X}$ has been defined then

$$
a^{*}= \begin{cases}b^{*} s_{n} & \text { if } s_{n} \in E_{X}, \\ b^{*} . s_{n} & \text { if } s_{n} \in F_{X}\end{cases}
$$

Let $\sim$ be the congruence on $F_{X} * E_{X}$ generated by the relation $\left\{\left(a a^{*}, a\right): a \in F_{X} * E_{X}\right\}$ and put $P_{X}=\left(F_{X} * E_{X}\right) / \sim$. It is shown in [10] that $P_{X}$ is the free right h-adequate semigroup on $X$. Further, every element of $P_{X}$ can be represented as $w_{0} A_{1} \ldots A_{n} w_{n}$, where each $A_{i}$ is in $E_{X}$, each $w_{i}$ is in $F_{X} \cup\{1\}$ with $w_{i} \neq 1$ when $i \neq 0, n$, and, for each $i$, $A_{i}<\left(w_{0} A_{1} \ldots w_{i-1}\right)^{*}$. An element of $P_{X}$ written in such a way is said to be in normal form.

From [10], we know that if $a \in P_{X}$ has normal form $w_{0} A_{1} \ldots A_{n} w_{n}$ then $a^{*}=A_{n}, w_{n}$ and thus we have the following lemma which is part of Proposition 3.3 of [10].

Lemma 2.1. Let $a, b$ be elements of $P_{X}$ with normal forms $w_{0} A_{1} \ldots A_{n} w_{n}$, $v_{0} B_{1} \ldots B_{m} v_{m}$ respectively. Then

$$
a \mathscr{L}^{*} b \text { if and only if } A_{n} \cdot w_{n}=B_{m} . w_{m} .
$$

On any right adequate semigroup there is a minimum left cancellative congruence $\sigma$. A description of $\sigma$ on right type A semigroups was given in Section 1. Now we want a characterisation of $\sigma$ on $P_{X}$. For any element $a=w_{0} A_{1} \ldots A_{k} w_{k}$ of $F_{X} * E_{X}$, we define $c(a)=w_{0} \ldots w_{k}$. If $a, b \in F_{X} * E_{X}$ and $a \sim b$ then $c(a)=c(b)$, so that we may regard $c$ as defined on $P_{X}$. Then $c$ is a ${ }^{*}$-homomorphism from $P_{X}$ onto $F_{X}^{1}$ and we have the following result.

Proposition 2.2 [10, Proposition 3.7]. On $P_{X}$,

(1) $(a, b) \in \sigma$ if and only if $c(a)=c(b)$ and

(2) $P_{X} / \sigma \cong F_{X}^{1}$.

The class $\mathscr{A}$ of right type A semigroups is contained in the class of right h-adequate semigroups and so, in the terminology of [15], $P_{X}$ is free for $\mathscr{A}$ over $X$, that is, $P_{X}$ is generated by $X$ and, for every $S \in \mathscr{A}$ and for every mapping $\alpha: X \rightarrow S$, there is a *-homomorphism $\beta: P_{X} \rightarrow S$ which extends $\alpha$. Since $\mathscr{A}$ is a quasi-variety, it follows from Lemma 4.112 of [15] that $P_{X} / \gamma$ is free in $\mathscr{A}$ over $\bar{X}=\{x \gamma: x \in X\}$, where $\gamma$ is the intersection of all the ${ }^{*}$-congruences $\rho$ on $P_{X}$ such that $P_{X} / \rho$ is right type $\mathrm{A}$. In other words, $\gamma$ is the minimum right type $\mathrm{A}^{*}$-congruence on $P_{X}$ and $P_{X} / \gamma$ is the free right type A semigroup on $\bar{X}$. As $|\bar{X}|=|X|$, we may regard $P_{X} / \gamma$ as the free right type A semigroup on $X$.

Our next task is to describe $\gamma$ on $P_{X}$. To help do this, we introduce the semigroup $A_{X}$ :

$$
A_{X}=\left\{(w, A) \in F_{X}^{1} \times E_{X}: w \leqslant a \text { for some } a \in A\right\}
$$

and the multiplication in $A_{X}$ is given by

$$
(w, A)(v, B)=(w v, A \cdot v \wedge B)
$$


It is an easy matter to verify that $A_{X}$ is a right type $\mathrm{A}$ semigroup with semilattice of idempotents $E\left(A_{X}\right)=\left\{(1, A): A \in E_{X}\right\}$ and with $(w, A)^{*}=(1, A)$.

Theorem 2.3. On $P_{X}, \gamma=\sigma \cap \mathscr{L}^{*}$ and $P_{X} / \gamma \cong A_{X}$.

Proof. Define a mapping $\theta: P_{X} \rightarrow A_{X}$ by putting $a \theta=\left(c(a), a^{*}\right)$ for each $a$ in $P_{X}$. Then $a^{*} \theta=\left(c\left(a^{*}\right), a^{*}\right)=\left(1, a^{*}\right)$ since $c$ maps all idempotents of $P_{X}$ to 1 . Thus $a^{*} \theta=(a \theta)^{*}$.

Next we note that if $C_{1}, \ldots, C_{k} \in E_{X}, u_{1}, \ldots, u_{k-1} \in F_{X}$ and $u_{k} \in F_{X}^{1}$ then, using the definition of $a^{*}$, an easy induction argument yields

$$
\left(C_{1} u_{1} \ldots C_{k} u_{k}\right)^{*}=\left(C_{1} \cdot\left(u_{1} \ldots u_{k}\right)\right)\left(C_{2} \cdot\left(u_{2} \ldots u_{k}\right)\right) \ldots\left(C_{k} \cdot u_{k}\right)
$$

Thus if $a, b \in P_{X}$ have normal forms $w_{0} A_{1} w_{1} \ldots A_{n} w_{n}, v_{0} B_{1} v_{1} \ldots B_{m} v_{m}$ respectively then

Hence

$$
\begin{aligned}
\left(a^{*} b\right)^{*} & =\left(\left(A_{n} \cdot w_{n}\right) v_{0} B_{1} v_{1} \ldots B_{m} v_{m}\right)^{*} \\
& =\left(\left(A_{n} \cdot w_{n}\right) \cdot\left(v_{0} \ldots v_{m}\right)\right)\left(B_{1} \cdot\left(v_{1} \ldots v_{m}\right)\right) \ldots\left(B_{m} \cdot v_{m}\right) \\
& =\left(\left(A_{n} \cdot w_{n}\right) \cdot c(b)\right)\left(B_{m} \cdot v_{m}\right) \\
& =a^{*} \cdot c(b) \wedge b^{*}
\end{aligned}
$$

$$
\begin{aligned}
(a b) \theta=\left(c(a b),(a b)^{*}\right) & =\left(c(a) c(b),\left(a^{*} b\right)^{*}\right) \\
& =\left(c(a) c(b), a^{*} \cdot c(b) \wedge b^{*}\right) \\
& =\left(c(a), a^{*}\right)\left(c(b), b^{*}\right) \\
& =(a \theta)(b \theta) .
\end{aligned}
$$

Thus $\theta$ is a ${ }^{*}$-homomorphism. Further, $\theta$ is surjective because if $(w, A) \in A_{X}$ then $w A$ is an element of $P_{X}$. Also $A \leqslant\{w\}$ since $w \leqslant a$ for some $a \in A$ and so $(w A)^{*}=A$. Hence $(w, A)=(w A) \theta$.

Thus $P_{X} / \operatorname{ker} \theta \cong A_{X}$, so that $\operatorname{ker} \theta$ is a right type A congruence and $\gamma \subseteq \operatorname{ker} \theta$. Now $(a, b) \in \operatorname{ker} \theta$ if and only if $c(a)=c(b)$ and $a^{*}=b^{*}$; so that, by Proposition 2.2, ker $\theta=\sigma \cap \mathscr{L}^{*}$.

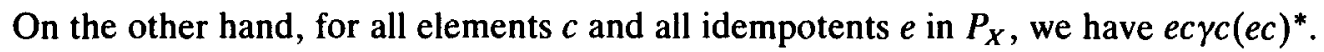
Repeated application of this to $A_{1} w_{1}, A_{2} w_{2},\left(A_{1} w_{1}\right)^{*} w_{2}$ etc., where $w_{0} A_{1} w_{1} \ldots A_{n} w_{n}$ is the normal form of an element $a$ of $P_{X}$, leads to

$$
a \gamma v_{0}\left(A_{1} v_{1}\right)^{*} \ldots\left(A_{n} v_{n}\right)^{*}
$$

where $v_{i}=w_{i} \ldots w_{n}$. Now $A_{i+1}<A_{i} \cdot w_{i}$ gives

$$
\left(A_{n} w_{n}\right)^{*}=A_{n} \cdot w_{n}<A_{i} \cdot v_{i}=\left(A_{i} v_{i}\right)^{*}
$$

for $i=1, \ldots, n-1$ and hence $a \gamma c(a) a^{*}$. Thus if $a, b$ are elements of $P_{X}$ with $c(a)=c(b)$ and $a^{*}=b^{*}$ then $a \gamma b$. In view of Proposition 2.2, we thus have $\sigma \cap \mathscr{L}^{*} \subseteq \gamma$ and so $\gamma=\sigma \cap \mathscr{L}^{*}$.

Corollary 2.4. $A_{X}$ is the free right type $A$ semigroup on $X$.

By comparing $A_{X}$ with the free objects in the category of SL2 $\delta$-semigroups as described in [1], we see that $A_{X}$ is the free SL2 $\delta$-semigroup on $X$. Thus, as noted in [3], we have the following corollary. 
Corollary 2.5. In the class of *-semigroups, the variety generated by the quasivariety of right type $A$ semigroups is the variety of SL2 $\delta$-semigroups.

Part (1) of the following result is an easy consequence of the definition of $\sigma$ on a right type A semigroup; part (2) follows from $(v, A)^{*}=(1, A)$.

Proposition 2.6. Let $(v, A),(w, B)$ be elements of $A_{X}$; then

(1) $(v, A) \sigma(w, B)$ if and only if $v=w$,

(2) $(v, A) \mathscr{L}^{*}(w, B)$ if and only if $A=B$.

CoROLlaRY 2.7. The free right type $A$ semigroup $A_{X}$ is proper.

We can now give an alternative proof of Theorem 3.3 of [8]. A homomorphism $\theta: S \rightarrow T$ of semigroups is called an $\mathscr{L}^{*}$-homomorphism in [8] if $a \theta=b \theta$ implies $a \mathscr{L}^{*} b$. When $S, T$ are right adequate, it is easy to see that if $\theta$ is an idempotent-separating *-homomorphism then $\theta$ is an $\mathscr{L}^{*}$-homomorphism. Thus the formulation in the next theorem does give Theorem 3.3 of [8].

THEOREM 2.8. Let $S$ be a right type A semigroup. Then $S$ is the image of a proper right type A semigroup under an idempotent-separating *-homomorphism.

Proof. Every right type A semigroup is a ${ }^{*}$-homomorphic image of some free right type A semigroup. Thus $S \cong A_{X} / \rho$ for some $X$ and some ${ }^{*}$-congruence $\rho$ on $A_{X}$. Now $\rho_{\min } \subseteq \rho$; so $A_{X} / \rho \cong\left(A_{X} / \rho_{\min }\right) /\left(\rho / \rho_{\min }\right)$. Since $A_{X}$ is proper, it follows from Proposition 1.7 that $A_{X} / \rho_{\min }$ is proper. Since $\rho\left|E\left(A_{X}\right)=\rho_{\min }\right| E\left(A_{X}\right)$, the ${ }^{*}$-congruence $\rho / \rho_{\min }$ is idempotent-separating and the result follows.

We remark that it is evident from the definition of $\rho_{\min }$ that $\rho_{\min } \subseteq \sigma$; so that, in the terminology of [1] and [2], $A_{X} / \rho_{\min }$ is quasi-free. A result similar to Theorem 2.8 is stated in [1] and [2], where it is asserted that every SL2 $\gamma$-semigroup is the image of a quasi-free SL2 $\gamma$-semigroup under an idempotent-separating ${ }^{*}$-homomorphism.

We also remark that Theorem 2.8 was proved in [16] by analysing *-congruences on $M$-semigroups and using the characterisation of $A_{X}$ as an $M$-semigroup.

3. Properties of $A_{X}$. The relation $\mathscr{R}^{*}$ on a semigroup is the dual of $\mathscr{L}^{*} ; \mathscr{D}^{*}$ is the join of $\mathscr{L}^{*}$ and $\mathscr{R}^{*}$. We describe the relation $\mathscr{R}^{*}$ on $A_{X}$ and show that $A_{X}$ is a single $\mathscr{D}^{*}$-class. By contrast, all Green's relations on $A_{X}$ are trivial. We then show that $A_{X}$ satisfies certain maximal conditions, has solvable word problem and is residually finite.

For any element $(v, A)$ of $A_{X}$, we define the subset $\operatorname{IS}(v, A)$ of $F_{X}^{1}$ by

$$
\operatorname{IS}(v, A)=\left\{u \in F_{X}^{1}: u v \in A\right\} \text {. }
$$

Since $(v, A)$ is a member of $A_{X}, v$ is a final segment of some element of $A$ and so $\operatorname{IS}(v, A)$ is not empty. Furthermore, since $A=\max A$, it is clear that $\operatorname{IS}(v, A)=\{1\}$ or $\operatorname{IS}(v, A)=\max \operatorname{IS}(v, A)$. In the latter case, $\operatorname{IS}(v, A)$ is a member of $E_{X}$.

Proposition 3.1. Let $(v, A),(w, B)$ be elements of $A_{X}$; then $(v, A) \mathscr{R}^{*}(w, B)$ if and only if $\operatorname{IS}(v, A)=\operatorname{IS}(w, B)$.

Proof. Put $H=\operatorname{IS}(v, A)$. Then $A=H . v \cup(A \backslash H . v)$. If $t \in A \backslash H . v$ then $t$ and $u v$ are incomparable for any element $u$ of $F_{X}$ since $A=\max A$. Hence we see that $A \backslash H . v$ is 
a subset of $C \cdot v \wedge A$ for any member $C$ of $E_{X}$. Thus, for $C, D \in E_{X}$, we have $C . v \wedge A=D \cdot v \wedge A$ if and only if $C . v \wedge H . v=D . v \wedge H . v$. If $H=\{1\}$, this latter condition is equivalent to $C . v=D . v$ and it is not difficult to see that $(v, A)$ is right cancellable. If $H \neq\{1\}$ then, since $F_{X}$ acts on $E_{X}$ by semilattice homomorphisms, we have $C . v \wedge A=D \cdot v \wedge A$ if and only if $(C \wedge H) \cdot v=(D \wedge H) \cdot v$, that is, if and only if $C \wedge H=D \wedge H$. It is now easy to see that $(v, A) \mathscr{R}^{*}(1, H)$ and the result follows.

Corollary 3.2. On $A_{X}$, the relation $\mathscr{D}^{*}$ is the universal relation, that is, $A_{X}$ is $\mathscr{D}^{*}$-simple.

Proof. Let $A \in E_{X}$ and let $v \in A$. Then $(v, A)$ is a member of $A_{X}$ and $\operatorname{IS}(v, A)=\{1\}$; so that $(v, A)$ is right cancellable. Since $(1, A) \mathscr{L}^{*}(v, A)$, it follows that every idempotent of $A_{X}$ is $\mathscr{L}^{*}$-related to a right cancellable element of $A_{X}$ and hence all idempotents of $A_{X}$ are in the same $\mathscr{D}^{*}$-class. The corollary now follows.

We now turn to Green's relations.

Proposition 3.3. On $A_{X}, \mathscr{J}=\iota$.

Proof. Suppose that $(v, A),(w, B)$ are $\mathscr{F}$-related. It follows that $v, w$ are subwords of each other and hence that $v=w$. Thus

$$
(v, A)=(1, C)(v, B)(1, D)
$$

for some $C, D \in E_{X}$; whence $A \leqslant B$. Similarly $B \leqslant A$ and so $(v, A)=(w, B)$.

As pointed out in [10], it is easy to see that the semilattice $E_{X}$ satisfies the ascending chain condition. We use this fact in the proof of the next result.

Proposition 3.4. $A_{X}$ satisfies the maximal condition for principal left ideals, for principal right ideals and for principal ideals.

Proof. Since $\mathscr{J}$ is trivial, it suffices to prove the condition for principal ideals. If $a_{1}, a_{2}, \ldots$ are the generators of an increasing sequence of principal ideals in $A_{X}$ then $a_{1} \sigma, a_{2} \sigma, \ldots$ are the generators of a similar sequence in $F_{X}$. Hence $a_{k} \sigma=a_{k+1} \sigma=\ldots$ for some $k$. Write $v=a_{k} \sigma$ and, for $j \geqslant k$, let $a_{j}=\left(v, A_{j}\right)$. It is easy to see that $A_{k} \leqslant A_{k+1} \leqslant$ ...; so that, for some positive integer $n, A_{n}=A_{n+1}=\ldots$ The result follows.

In [10], it is shown that an element of the free *-semigroup on $X$ can be effectively reduced to a normal form in $P_{X}$. Given an element $a$ of $P_{X}$ in normal form, it is clear that there is a finite procedure for finding $c(a)$ and $a^{*}$. We can determine in an effective way whether two elements of $F_{X}^{1}$ (resp. $E_{X}$ ) are equal and so, by virtue of Lemma 2.1, Proposition 2.2 and Theorem 2.3, we can decide when two elements in normal form in $P_{X}$ are related by the congruence $\gamma$. The following result is an immediate consequence.

Proposition 3.5. The word problem for $A_{X}$ is solvable.

We conclude this section by considering the residual finiteness of $A_{X}$.

Proposition 3.6. $A_{X}$ is residually finite in the class of right type $A$ semigroups.

Proof. Let $(v, A),(w, B)$ be distinct elements of $A_{X}$ and let $Y$ be the set of letters (elements of $X$ ) occurring in words in $A \cup B$. Let $Q$ be the set of elements $C$ of $E_{X}$ for 
which an element of $X \backslash Y$ occurs in a word belonging to $C$. It is clear that $Q$ is an ideal of $E_{X}$. It follows that

$$
J=\left\{(u, C) \in A_{X}: X \in Q\right\}
$$

is an ideal of $A_{X}$ and it is clear that it is in fact a *-ideal.

Next we let $k-1$ be the length of the longest word in $A \cup B$ and let $J_{k}$ be the ideal of $E_{X}$ consisting of all members of $E_{X}$ which contain a word of length at least $k$. Then

$$
K=\left\{(u, C) \in A_{X}: C \in J_{k}\right\}
$$

is a *-ideal of $A_{X}$. Now let $I=J \cup K$. Then $I$ is a *-ideal of $A_{X}$; so that, by Proposition 1.4, $A_{X} / I$ is a right type A semigroup and the natural homomorphism $v$ from $A_{X}$ onto $A_{X} / I$ is $a^{*}$-homomorphism. Since neither $(v, A)$ nor $(w, B)$ is a member of $I$, we have $(v, A) v \neq(w, B) v$. However, it is easy to see that $A_{X} / I$ is finite.

We recall that an algebra is hopfian if all its surjective endomorphisms are automorphisms. It is pointed out in [10] that the general result of Evans [6] that a finitely generated residually finite algebra in a variety of algebras is hopfian applies equally well to a quasi-variety of algebras. The following corollary is therefore immediate from Proposition 3.6.

Corollary 3.7. For a finite set $X, A_{X}$ is hopfian.

4. Sets of free generators. For a subset $Y$ of a right adequate semigroup $S$, we denote by $\langle Y\rangle^{*}$ the *-subsemigroup generated by $Y$. As we observed in Section 1 , if $S$ is right type $\mathrm{A}$ then so is $\langle Y\rangle^{*}$. If the inclusion map $Y \rightarrow\langle Y\rangle^{*}$ extends to an isomorphism of $A_{Y}$ onto $\langle Y\rangle^{*}$ then we say that $Y$ is a set of free generators for $\langle Y\rangle^{*}$.

The results of this section are inspired by the corresponding ones of Reilly [19] as were those of Section 4 in [10]. We start observing that $A_{X}$ has only one set of free generators.

Propostrion 4.1. Let $X$ be a non-empty set. Then the subset $\bar{X}=\{(x,\{x\}): x \in X\}$ is the only set of free generators for $A_{X}$.

Proof. For any subset $Y$ of $A_{X}$, let $Y_{r}=\left\{y^{*}: y \in Y\right\}$. Then $\bar{X}_{r}=\{(1,\{x\}): x \in X\}$ is the set of maximal elements of $E\left(A_{X}\right)$. We can thus characterize $\bar{X}$ as the unique subset of $A_{X}$ such that $\bar{X} \cap \bar{X}_{r}=\varnothing$ and $\bar{X}_{r}$ is the set of maximal elements of $E\left(A_{X}\right)$.

For any right type A semigroup $S$, we define the relation $\leqslant$ by

$$
a \leqslant b \text { if and only if } a=b a^{*} .
$$

As noted in [1] and [5], " $a=b a$ " " is equivalent to " $a=b e$ for some idempotent $e$ in $S$ " and the relation $\leqslant$ is a compatible partial order on $S$ which extends the natural order of the semilattice of idempotents of $S$. It will always be clear from the context when $\leqslant$ is being used for the relation just described and when it is the partial order on $F_{X}$ used throughout the paper.

We now give criteria for a subset of a right type A semigroup to be a set of free generators for the ${ }^{*}$-subsemigroup which it generates. 
Proposition 4.2. Let $Y$ be subset of a right type A semigroup S. Then $Y$ is a set of free generators for $\langle Y\rangle^{*}$ if and only if

(1) no pair of elements of $\langle Y\rangle$ have a lower bound in $\langle Y\rangle^{*}$, and

(2) if $\left(y_{t} \ldots y_{1}\right)^{*} \geqslant \prod_{j=1}^{m}\left(y_{j p(j)} \ldots y_{j 1}\right)^{*}$, where the $y_{i}$ and $y_{j k}$ are elements of $Y$, then there is a $j \in\{1, \ldots, m\}$ such that $y_{i}=y_{j i}$ for $i=1, \ldots, t$.

Proof. Let $X$ be a set in one-one correspondence with $Y$ and let $\theta: X \rightarrow Y$ be a bijection. Then there is a unique ${ }^{*}$-homomorphism $\psi$ from $A_{X}$ onto $\langle Y\rangle^{*}$ such that $(x,\{x\}) \psi=x \theta$ for each $x \in X$. Thus $Y$ is a set of free generators for $\langle Y\rangle^{*}$ if and only if $\psi$ is injective.

Suppose first that $\psi$ is injective. We identify $\bar{X}$ with $Y$ and $A_{X}$ with $\langle Y\rangle^{*}$. A typical element of $\langle\bar{X}\rangle$ is $(v,\{v\})$, where $v \in F_{X}$, and it is easily seen that condition (1) holds. If the hypothesis of condition (2) holds then we have

$$
\left(1,\left\{x_{t} \ldots x_{1}\right\}\right) \geqslant \prod_{j=1}^{m}\left(1,\left\{x_{j p(j)} \ldots x_{j 1}\right\}\right) ;
$$

so that $x_{t} \ldots x_{1}$ is a final segment of $x_{j p(j)} \ldots x_{j 1}$ for some $j$. Since $F_{X}$ is the free semigroup on $X$, we have $x_{i}=x_{j i}$ for $i=1, \ldots, t$ and the conclusion of condition (2) now follows.

Now suppose that conditions (1) and (2) hold and let $A, B \in E_{X}$ be such that $(1, A) \psi=(1, B) \psi$. Let $A=\left\{v_{1}, \ldots, v_{m}\right\}$ and $B=\left\{w_{1}, \ldots, w_{n}\right\}$. Then, since $A=\max A$, $B=\max B$, no two of $v_{1}, \ldots, v_{m}$ are comparable and no two of $w_{1}, \ldots, w_{n}$ are comparable. Further,

$$
\begin{aligned}
& (1, A)=\left(1,\left\{v_{1}\right\}\right) \ldots\left(1,\left\{v_{m}\right\}\right)=\left(v_{1},\left\{v_{1}\right\}\right)^{*} \ldots\left(v_{m},\left\{v_{m}\right\}\right)^{*}, \\
& (1, B)=\left(1,\left\{w_{1}\right\}\right) \ldots\left(1,\left\{w_{n}\right\}\right)=\left(w_{1},\left\{w_{1}\right\}\right)^{*} \ldots\left(w_{n},\left\{w_{n}\right\}\right)^{*} .
\end{aligned}
$$

Thus $(1, A) \psi=(1, B) \psi \leqslant\left(1,\left\{w_{1}\right\}\right) \psi=\left(\left(w_{1},\left\{w_{1}\right\}\right) \psi\right)^{*}$. For each $j=1, \ldots, m$, let $v_{j}=$ $x_{j p(j)} \ldots x_{j 1}$, where $x_{j k} \in X$ for $1 \leqslant k \leqslant p(j)$, and let $w_{1}=x_{t} \ldots x_{1}$, where $x_{1}, \ldots, x_{t} \in X$. For each $x_{j k}$ and each $x_{i}$, put $x_{j k} \theta=y_{j k}$ and $x_{i} \theta=y_{i}$. Then

and

$$
\left(y_{t} \ldots y_{1}\right)^{*}=\left(\left(w_{1},\left\{w_{1}\right\}\right) \psi\right)^{*}=\left(1,\left\{w_{1}\right\}\right) \psi
$$

Hence

$$
\prod_{j=1}^{m}\left(y_{j p(j)} \ldots y_{j 1}\right)^{*}=\prod_{j=1}^{m}\left(\left(v_{j},\left\{v_{j}\right\}\right) \psi\right)^{*}=(1, A) \psi
$$

$$
\left(y_{t} \ldots y_{1}\right)^{*} \geqslant \prod_{j=1}^{m}\left(y_{j p(j)} \ldots y_{j 1}\right)^{*}
$$

and so, by condition (2), there is some $j$ in $\{1, \ldots, m\}$ such that $y_{i}=y_{j i}$ for $i=1, \ldots, t$. It follows that $x_{i}=x_{j i}$ for $i=1, \ldots, t$ and so $w_{1}$ is a final segment of some $v_{j}$. A similar argument shows that $v_{j}$ is a final segment of some $w_{k}$. Now we have that $w_{1}$ and $w_{k}$ are comparable so that $k=1$ and $w_{1}=v_{j}$. Replacing $w_{1}$ by $w_{i}$ shows that each $w_{i}$ is some $v_{j}$; similarly each $v_{j}$ is some $w_{i}$. Thus $A=B$ and $\psi$ is idempotent-separating.

Now let $(v, A),(w, B)$ be elements of $A_{X}$ with $(v, A) \psi=(w, B) \psi$. Since $\psi$ is a *-homomorphism, $(1, A) \psi=(1, B) \psi$ and so $A=B$. Hence

$$
\begin{aligned}
(v,\{v\}) \psi(1, A) \psi & =(v, A) \psi=(w, A) \psi \\
& =(w,\{w\}) \psi(1, A) \psi
\end{aligned}
$$


so that $(v,\{v\}) \psi,(w,\{w\}) \psi$ have a lower bound in $\langle Y\rangle^{*}$. It follows from condition (1) that $(v,\{v\}) \psi=(w,\{w\}) \psi$ and, since $\psi$ is a ${ }^{*}$-homomorplism, we obtain $(1,\{v\}) \psi=$ $(1,\{w\}) \psi$. As $\psi$ is idempotent separating, we conclude that $v=w$ and hence $\psi$ is injective.

Let $\bar{F}_{X}=\left\{(w,\{w\}): w \in F_{X}\right\}$. We now consider which subsets of $\bar{F}_{X}$ are sets of free generators for the *-subsemigroups of $A_{X}$ which they generate. It is easy to see that any subset of $\bar{F}_{X}$ satisfies condition (1) of Proposition 4.2. Thus for a subset $Y$ of $\bar{F}_{X}$, condition (2) is necessary and sufficient for $Y$ to be a set of free generators for $\langle Y\rangle^{*}$. We recall that a subset $C$ of $F_{X}$ is a suffix code over $X$ if $F_{X} C \cap C=\varnothing$. We refer the reader to Chapter 5 of [13] for the essential facts about suffix codes.

Corollary 4.3. Let $Y$ be a subset of $\bar{F}_{X}$ and let $C=\left\{w \in F_{X}:(w,\{w\}) \in Y\right\}$. Then the *-subsemigroup $\langle Y\rangle^{*}$ of $A_{X}$ is freely generated by $Y$ if and only if $C$ is a suffix code over $X$.

Proof. Suppose that $\langle Y\rangle^{*}$ is freely generated by $Y$. If $w \in F_{X} C \cap C$ then $w$ has a proper final segment, say $u$, in $C$. Let $y=(w,\{w\}), y^{\prime}=(u,\{u\})$; then $\left(y^{\prime}\right)^{*}>y^{*}$, contradicting condition (2). Hence $F_{X} C \cap C=\varnothing$ and $C$ is a suffix code over $X$.

Now let $C$ be a suffix code over $X$. As remarked above, we need only prove that condition (2) holds. Suppose then that

$$
\left(y_{t} \ldots y_{1}\right)^{*} \geqslant \prod_{j=1}^{m}\left(y_{j p(j)} \ldots y_{j 1}\right)^{*}
$$

Let $y_{i}=\left(w_{i},\left\{w_{i}\right\}\right)$ for $i=1, \ldots, t$ and $y_{j k}=\left(w_{j k},\left\{w_{j k}\right\}\right)$ for $j=1, \ldots, m$ and $k=$ $1, \ldots, p(j)$; so that $w_{i}$ and $w_{j k}$ are in $F_{X}$. Then our assumption is

$$
\left\{w_{t} \ldots w_{1}\right\} \geqslant \max \left\{w_{1 p(1)} \ldots w_{11}, \ldots, w_{m p(m)} \ldots w_{m 1}\right\}
$$

and so $w_{t} \ldots w_{1}$ is a final segment of $w_{j p(j)} \ldots w_{j 1}$ for some $j$. Thus either $w_{1}=w_{j 1}$ or one of $w_{1}, w_{j 1}$ is a proper final segment of the other. The latter is impossible since $w_{1}, w_{j 1} \in C$ and $C$ is a suffix code. Hence $w_{1}=w_{j 1}$ and so $w_{t} \ldots w_{2}$ is a final segment of $w_{j p(j)} \ldots w_{j 2}$. We now get $w_{2}=w_{j 2}$ and similarly we obtain $w_{i}=w_{j i}$ for $i=1, \ldots, t$. Thus $y_{i}=y_{j i}$ for $i=1, \ldots, t$ and condition (2) holds as required.

Over sets with at least two elements there are infinite suffix codes and so we have the following immediate consequence of Corollary 4.3.

CorollarY 4.4. If $2 \leqslant|X|$ then there is a countably infinite subset $Y$ of $\bar{F}_{X}$ such that $Y$ is a set of free generators for the *-subsemigroup $\langle Y\rangle^{*}$ of $A_{X}$.

Since a right type A semigroup $S$ is right $h$-adequate we know by Lemma 4.5 of [10] that if $S=\langle a\rangle^{*}$ for some $a$ in $S$ then $E(S)=\left\{\left(a^{k}\right)^{*}: k \in \mathbb{Z}, k \geqslant 1\right\}$. In particular, $E(S)$ is a chain, so that if $s, t$ are any two elements of $S$ then $s, t$ cannot freely generate $\langle s, t\rangle^{*}$ because $E\left(\langle s, t\rangle^{*}\right)$ is not isomorphic to $E\left(A_{\{x, y\}}\right)$. Thus if $|Z|=1$ then $A_{Z}$ does not contain copies of $A_{X}$ for any set $X$ with $2 \leqslant|X|$. We conclude this section by showing that a non-idempotent element $a$ in any $A_{X}$ freely generates $\langle a\rangle^{*}$.

Proposition 4.5. If $(v, A) \in A_{X}$ and $v \neq 1$ then $(v, A)$ is a free generator of $\langle(v, A)\rangle^{*}$.

Proof. If $(v, A)^{t}(1, B)=(v, A)^{k}(1, C)$ for any $B, C \in E_{X}$ and positive integers $t, k$ then $v^{t}=v^{k}$; so that $t=k$ and condition (1) of Proposition 4.2 holds. 
Let $a=(v, A)$ and suppose that

$$
\left(a^{t}\right)^{*} \geqslant \prod_{j=1}^{m}\left(a^{p(j)}\right)^{*}
$$

Then $t \leqslant \max \{p(1), \ldots, p(m)\}$ and hence condition (2) of Proposition 4.2 holds.

5. Free right type $A$ semigroups with central idempotents. Let $\mathscr{B}$ be the class of right type A semigroups with central idempotents. From [7], we know that if $S \in \mathscr{B}$ then $S$ is a strong semilattice of left cancellative monoids. In this section we describe the free objects in $\mathscr{B}$. On any right type A semigroup $S$ there is a ${ }^{*}$-congruence $v$ which is minimum among those *-congruences $\rho$ such that $S / \rho$ has central idempotents. As $\mathscr{B}$ is a quasi-variety of ${ }^{*}$-semigroups and is contained in the quasi-variety of right type $\mathrm{A}$ semigroups, it follows (as in Section 2) from Lemma 4.112 of [15] that $A_{X} / v$ is the free object in $\mathscr{B}$ on $X$. To obtain a more explicit description, we begin by considering some properties of $v$.

For a word $w$ in $F_{X}$, we define alph(w) to be the subset of $X$ consisting of those elements which actually occur in $w$. For a subset $A$ of $F_{X}$ we define

$$
\operatorname{alph}(A)=\bigcup\{\operatorname{alph}(w): w \in A\} .
$$

Lemma 5.1. For each $w \in F_{X},(1,\{w\}) v(1, \operatorname{alph}(w))$.

Proof. We use induction on the length of $w$. Assuming the result for words of length $n$, let $w=x_{1} \ldots x_{n+1}$.

Then $\left(x_{n+1}, \operatorname{alph}(w)\right) \in A_{X}$ and

$$
\begin{aligned}
\left(x_{n+1}, \operatorname{alph}(w)\right)= & \left(x_{n+1},\left\{x_{n+1}\right\}\right)\left(1, \operatorname{alph}\left(x_{1} \ldots x_{n}\right)\right) \\
& v\left(1, \operatorname{alph}\left(x_{1} \ldots x_{n}\right)\right)\left(x_{n+1},\left\{x_{n+1}\right\}\right) \\
& v\left(1,\left\{x_{1} \ldots x_{n}\right\}\right)\left(x_{n+1},\left\{x_{n+1}\right\}\right) \\
& =\left(x_{n+1},\left\{x_{1} \ldots x_{n+1}\right\}\right) \\
& =\left(x_{n+1},\{w\}\right) .
\end{aligned}
$$

As $v$ is a *-congruence, we thus have $(1, \operatorname{alph}(w)) v(1,\{w\})$ and the result follows.

Corollary 5.2. If $(1, A) \in A_{X}$ then $(1, A) v(1, \operatorname{alph}(A))$.

Proof. Let $A=\left\{w_{1}, \ldots, w_{m}\right\}$. Then, by Lemma 5.1,

$$
(1, A)=\left(1,\left\{w_{1}\right\}\right) \ldots\left(1,\left\{w_{m}\right\}\right) v\left(1, \operatorname{alph}\left(w_{1}\right)\right) \ldots\left(1, \operatorname{alph}\left(w_{m}\right)\right)=(1, \operatorname{alph}(A)) .
$$

Corollary 5.3. If $(w, A),(w, B) \in A_{X}$ and $\operatorname{alph}(A)=\operatorname{alph}(B)$ then $(w, A) v(w, B)$.

Proof. Since $(w, A)=(w,\{w\})(1, A)$ and $(w, B)=(w,\{w\})(1, B)$, we need only show that $(1, A) v(1, B)$. But this follows from Corollary 5.2 since alph $(A)=\operatorname{alph}(B)$.

For a non-empty set $X$, we denote the free semilattice with identity on $X$ by $Y_{X}$. The elements of $Y_{X}$ are all finite subsets of $X$ and the operation is set-theoretic union. We define a subsemigroup $S_{X}$ of the direct product $F_{X}^{1} \times\left(Y_{X} \backslash\{1\}\right)$ as follows:

$$
S_{X}=\left\{(w, T) \in F_{X}^{1} \times\left(Y_{X} \backslash\{1\}: \operatorname{alph}(w) \subseteq T\right\}\right.
$$

where $\operatorname{alph}(1)=\varnothing$.

It is easy to verify that $S_{X}$ is right type A with central idempotents. 
Proposition 5.4. The mapping $\psi: A_{X} \rightarrow S_{X}$ defined by

$$
(w, A) \psi=(w, \operatorname{alph}(A))
$$

is a surjective *-homomorphism and $\operatorname{ker} \psi=v$. so

Proof. Let $(w, A),(v, B) \in A_{X}$. Then $v$ is a final segment of some member of $B$ and

$$
\operatorname{alph}(A \cdot v \wedge V)=\operatorname{alph}(A \cup B)=\operatorname{alph}(A) \cup \operatorname{alph}(B) .
$$

It is now easy to see that $\psi$ is a ${ }^{*}$-homomorphism. Further if $(w, T) \in S_{X}$ then $(w, A) \in A_{X}$, where

$$
A=\{w\} \cup(T \backslash \operatorname{alph}(w))
$$

and $(w, A) \psi=(w, T)$; so that $\psi$ is surjective.

Thus ker $\psi$ is a ${ }^{*}$-congruence and $A_{X} /$ ker $\psi$ is right type A with central idempotents; so that $v \subseteq \operatorname{ker} \psi$.

If $((w, A),(w, B)) \in \operatorname{ker} \psi$ then $w=v$ and alph $(A)=\operatorname{alph}(B)$ so that, by Corollary 5.3 , $\operatorname{ker} \psi \subseteq v$. Thus $v=\operatorname{ker} \psi$ as required.

COROLlary 5.5. The *-semigroup $S_{X}$ is the free object in $\mathscr{B}$ on $X$.

We conclude by noting that we can represent $S_{X}$ as a strong semilattice of free monoids as follows:

$$
S_{X} \cong \bigcup_{A \in E} F_{A}^{1},
$$

where $E=Y_{X} \backslash\{1\}$ and the linking homomorphisms $\phi_{A, B}: F_{A}^{1} \rightarrow F_{B}^{1}$ are simply the inclusion mappings for $A \subseteq B$.

\section{REFERENCES}

1. A. Batbedat, $\gamma$-demi-groups, demi-modules, produit demi-directs, Semigroups, Proceedings, Oberwolfach, Germany, 1978, Lecture Notes in Mathematics 855 (Springer, 1981), $1-18$.

2. A. Batbedat, Les demi-groupes idunaires ou gamma-demi-groupes, Cahiers Mathématiques Montpellier 20 (Montpellier, 1981).

3. A. Batbedat and J. B. Fountain, Connections between left adequate semigroups and $\gamma$-semigroups, Semigroup Forum 22 (1981), 59-65. 434-451.

4. A. H. Clifford, The free completely regular semigroup on a set, J. Algebra 59 (1979),

5. A. El-Qallali, Structure theory for abundant and related semigroups, D. Phil. thesis, University of York, 1980.

6. T. Evans, Finitely presented loops, lattices, etc. are hopfian, J. London Math. Soc. 44 (1969), 551-552.

7. J. B. Fountain, Right PP monoids with central idempotents, Semigroup Forum 13 (1977), 229-237. $285-300$

8. J. B. Fountain, a class of right PP monoids, Quart. J. Math. Oxford. Ser. (2) 28 (1977),

9. J. B. Fountain, Adequate semigroups, Proc. Edinburgh Math. Soc. 22 (1979), 113-125.

10. J. B. Fountain, Free right h-adequate semigroups, Semigroups, theory and applications, Lecture Notes in Mathematics 1320 (Springer, 1988), 97-120. 

26-38.

11. A. Horn and N. Kimura, The category of semilattices, Algebra Universalis 1 (1971),

12. M. V. Lawson, The structure of type A semigroups, Quart. I. Math. Oxford Ser. (2) 37 (1986), 279-298.

13. G. Lallement, Semigroups and combinatorial applications (Wiley, 1979).

14. D. B. McAlister, One-to-one partial right translations of a right cancellative semigroup, $J$. Algebra 43 (1976), 231-251.

15. R. N. McKenzie, G. F. McNulty and W. Taylor, Algebras, lattices, varieties, vol. 1 (Wadsworth, 1987).

16. A. Palmer, Proper right type A semigroups, M. Phil. thesis, University of York, 1982.

17. F. Pastijn, A representation of a semigroup by a semigroup of matrices over a group with zero, Semigroup Forum 10 (1975), 238-249.

18. M. Petrich, Inverse semigroups (Wiley, 1984).

19. N. R. Reilly, Free generators in free inverse semigroups, Bull. Austral. Math. Soc. 7 (1972), 407-424.

20. H. E. Scheiblich, Free inverse semigroups, Proc. Amer. Math. Soc. 38 (1973), 1-7.

Department of Mathematics

UNIVERSITY OF YORK

HESLINGTON

YORK YO1 5DD 